Goldschmidt 2021 Abstract

https://doi.org/10.7185/gold2021.3322

\section{Spatial distribution and speciation of Rare Earth Elements in mine tailings from ion-adsorption deposits in Southern China}

\author{
NOÉMIE JANOT ${ }^{1,2,3}$, HERMINE HUOT ${ }^{2,4}$, CAMILLE \\ RIVARD $^{5,6}$, SHARON BONE $^{7}$, YE-TAO TANG ${ }^{8}$, \\ FRANÇOISE WATTEAU ${ }^{1}$ AND EMMANUELLE \\ MONTARGES-PELLETIER ${ }^{2}$
}

${ }^{1}$ INRAE/Université de Lorraine

${ }^{2} \mathrm{CNRS} /$ Université de Lorraine

${ }^{3}$ INRAE/Bordeaux Sciences Agro

${ }^{4}$ Sun-Yat Sen University

${ }^{5}$ Synchrotron SOLEIL

${ }^{6}$ INRAE TRANSFORM

${ }^{7} \mathrm{SSRL}$

${ }^{8}$ Sun Yat-sen University

Presenting Author: noemie.janot@inrae.fr

Rare Earth Elements (REEs) are essential components of many high-tech products. In industrial materials, REEs come at $90 \%$ from Southern China ion-adsorption deposits, from which they are recovered by heap leaching using ammonium sulfate. Such large-scale process leads to severe environmental damage, and produces large tailings. Elucidating the nature of REE-bearing phases in these mine tailings is critical for predicting REE dynamics and designing a successful sustainable reclamation strategy, phyto-stabilization being currently tested.

First investigations on the tailings soils revealed their high heterogeneous mineral composition, supporting the need for micro-scale investigation. XRD and TEM-EDXS identified quartz, K-feldspars and kaolinite as major phases, and optical microscope observations evidenced Fe-rich weathered layers surrounding particles, as well as organic material that might play a role in REEs distribution.

$\mathrm{X}$-ray spectromicroscopy analyses of soil thin sections (bare and planted) were performed at SSRL (beamline 2-3) and SOLEIL (LUCIA). We targeted the most concentrated REEs in the bulk soil, i.e., La, Ce and Nd. Overlaps in the fluorescence lines of REEs and transition metals (Ba, Ti) obliged us to perform fitting of the fluorescence signal in each pixel of the maps. Then, $\mu$ XANES spectra at REE Ledges were collected to confirm the presence of REEs. The use of $\mu$ XANES allowed us to detect very low quantities of REEs that were not detectable in global fluorescence maps. The combination of spectromicroscopy and fluorescence mapping was crucial and powerful to detect low levels of REEs in heterogeneous material.

We evidenced the co-localization of REEs with $\mathrm{Fe}$ oxides, and, in reclaimed and vegetated samples, with roots. The high number of measurements performed on LUCIA beamline showed us that Ce speciation was predominantly $\mathrm{Ce}^{4+}$ when not accompanied by others REEs. In contrast, $\mathrm{Ce}^{3+}$ is systematically associated with $\mathrm{Nd}^{3+}$ and $\mathrm{La}^{3+}$.

This work is, to our knowledge, the first to address REE distribution and speciation in soils. It provides significantly enhanced understanding of processes governing REE behaviour in mine tailings and will help designing successful reclamation techniques of contaminated land. It will contribute to validate process-based geochemical models describing REE behaviour, allowing to predict REE fate with ongoing reclamation. 